\title{
¿Es posible la formación de personas éticas y comprometidas en la universidad? La asignatura de Educación para la paz y la igualdad en los Grados de Magisterio
}

\author{
Luis Torrego Egido (*), Suyapa Martínez Scott $\left(^{*}\right)$ y Miriam Sonlleva Velasco (*)
}

(*) Universidad de Valladolid - España

\section{RESUMEN}

La universidad debería formar estudiantes bien informados, dotados de sentido crítico y comprometidos con la transformación social desde una ética universal basada en la solidaridad y en el bien común. Sin embargo, en la universidad actual predomina una visión utilitarista de la formación. La asignatura Educación para la paz y la igualdad se imparte en los Grados de Educación Infantil y Educación Primaria de la Universidad de Valladolid. Su finalidad es contribuir a la formación de personas éticas y comprometidas. En este artículo se analizan la potencialidad y los logros de esa asignatura. Mediante un estudio de caso en el que se utilizan entrevistas, observación no participante, cuestionarios, análisis de documentos y análisis de producciones del alumnado de la asignatura, se evalúa la valoración por parte de los estudiantes y la influencia que ejerce en ellos, así como los aprendizajes que genera. Se constata una mayor sensibilización, una mejora en la comprensión de la realidad global y el descubrimiento de estrategias didácticas para trabajar la paz y la igualdad en la escuela, pero sigue existiendo una actitud pasiva en buena parte del alumnado. Se precisa una educación contra el conformismo y la indiferencia.

Palabras Clave: Formación inicial, Compromiso ético, Estudio de caso, Modelos de universidad.

\section{Is it possible to educate ethical and committed people in the university? The subject of Education for peace and equality in the Degrees of Teaching}

\begin{abstract}
The university should educate well-informed students, endowed with critical sense and committed to social transformation from a universal ethic based on solidarity and the common good. However, at the current university, a utilitarian vision of education predominates. The subject Education for peace and equality is been taught in the Degrees of Early Childhood Education and Primary Education at the University of Valladolid. Its purpose is contributing to educate ethical and committed people. This paper analyzes the potentiality and achievements of this subject. Through a case study using interviews, non-participant observation, questionnaires, analysis of documents and that of student productions for the subject, assessment made by the students and their influence on them are evaluated, as well as the learnings generated by it. There is a greater awareness, an improvement in the understanding of the global reality and the discovery of didactic strategies to work for peace and equality at school, but a passive attitude remains in many of the students. An education against conformism and indifference is needed.
\end{abstract}

Keywords: Initial formation, Ethical commitment, Case study, University models.

\section{Introducción}

La UNESCO atribuye a la universidad la obligación de formar estudiantes bien informados, dotados de sentido crítico que les haga capaces de analizar los problemas sociales, así como de generar alternativas, aplicarlas y asumir responsabilidades sociales (UNESCO, 1998). Hoy, la universidad está lejos de cumplir esas finalidades; en su lugar, el contexto neoliberal y positivista que la ha penetrado ha logrado que no se discutan los fines a los que se encamina la institución, que aparecen impregnados de la lógica de la competitividad y del eficientismo. Dicen los economistas que se pierde también cuando se tiene una oportunidad de obtener beneficio y no se aprovecha. Desde esa perspectiva, como señala Manzano, la universidad es la institución más perdedora que pueda imaginarse (Manzano, 2011, p. 265).

Dentro de las visiones de la universidad y de su función hay una que la considera como una institución autónoma, que desarrolla desde esa autonomía su función de generar y transmitir 
conocimiento para la formación integral de personas, científica e intelectualmente capaces. Hoy, sin embargo, podríamos calificar como tradicional a ese enfoque, pues predomina otro que exige el control de la universidad y cree que su función es desarrollar, con eficiencia, conocimientos relevantes para el tejido económico y para la mejora de la competitividad del sistema productivo (Manzano y Torrego, 2009).

Se ha extendido, así, una perspectiva reduccionista de la educación superior que ha llegado también a los organismos inter y supranacionales, de modo que desde ellos se la atribuye el objetivo de impulsar una economía del conocimiento que mejore la competitividad (Consejo de la Unión Europea, 2007) o de mejora de la productividad económica y de la eficiencia laboral y productiva de los países (Banco Mundial, 2003; OCDE, 2007) o se la considera, casi exclusivamente, como un producto. Esa visión, colonizada por la economía, conduce a visiones simplificadas de la calidad en la docencia y de los objetivos de esta.

Sin embargo, se abre paso otro modelo que, rescatando la idea de la formación integral, subraya el compromiso de la universidad con la consecución de una sociedad más justa y de un planeta sostenible. Ese modelo dispone, también, del aval internacional, como el recogido en la ya citada Declaración Mundial sobre la Educación Superior en el siglo XXI (UNESCO, 1998), a la que se adhirieron los gobiernos de más de 180 países o la Declaración de Talloires sobre las responsabilidades cívicas sociales y las funciones cívicas de la educación superior, firmada, en 2005, por 27 universidades de todo el mundo en esa ciudad francesa. Este último modelo, certeramente titulado como "la Universidad como debiera ser" (Boni y Gaspers, 2011), pues está lejos de plasmarse como una corriente mayoritaria en la realidad académica, promueve una educación que genere el compromiso cívico, la acción colectiva y la dimensión solidaria en la formación de las personas.

La sobreorientación economicista a la que antes aludíamos ha llegado también a nuestros planes de estudio que no sólo son el resultado de una lucha de intereses de grupos académicos, estructurados en departamentos, sino que también son el producto de un énfasis de la utilidad, entendida en términos casi exclusivamente mercantiles, que invoca la profesionalización y entiende la misma como el desarrollo de competencias técnicas en una lógica meramente instrumentalista (Calvo, Rodríguez-Hoyos y Haya, 2015).

La consecuencia de este proceso es que se genera una formación que contribuye a constituir estudiantes que responden a una lógica extendida: personas que se tienden a concentrar en sus fines privados, fundamentalmente económicos, creando así una base orientada por el utilitarismo y la falta de compromiso con valores humanizadores y de justicia.

Es preciso avanzar en sentido contrario, es decir en la formación de personas orientadas hacia esa ética planetaria descrita por Leonardo Boff (2001), fundada en la solidaridad, en la compasión y la liberación, en la justicia, en la dignidad de la Tierra, en los cuidados, en lo que él denomina utilitarismo social, presidida por una nueva racionalidad que logre unas prácticas que proporcionen la mayor felicidad y realización para el mayor número posible de personas. Y, para ello, la universidad puede ser un instrumento privilegiado.

Esa formación ética y comprometida se presenta como una necesidad por numerosos autores y se ha identificado a la universidad como una entidad que ha de asumir esa responsabilidad, tanto en contextos europeos como americanos. Algunas de esas voces abordan el asunto aproximándose desde la educación ética y cívica (Bolívar, 2005; Jover, López y Quiroga, 2011; Martínez, Buxarrais y Esteban, 2002; Martínez-Martín, 2006; Muhr, 2010; Petrova, 2010) y otras lo hacen, señalando en primer lugar la formación en el compromiso social (Aguilar, 2013; Cecchi, Pérez y Sanllorenti, 2013; Del Río y Celorio, 2018; Ibarrola-García y Artuch, 2016; Manzano, 2011; Vallaeys, 2014).

Es cierto que no hay una coincidencia absoluta en estas formulaciones de la responsabilidad de la universidad y en las características concretas que debe caracterizar a esta formación, pero sí puede establecerse un acuerdo muy generalizado en torno a la función de la universidad en el desarrollo en sus estudiantes de capacidades para participar, activamente y con una visión transformadora, en la vida política y social de sus comunidades, con una mirada cosmopolita, y para que en ellos se despliegue el compromiso con la justicia social.

Por otra parte, los estudios que analizan propuestas didácticas de esa formación ética y ciudadana son escasas o se centran en ámbitos parciales. Así, aparecen investigaciones evaluativas centradas en la formación proporcionada por el aprendizaje-servicio en la universidad (Santos, Sotelino y Lorenzo, 2016), en el análisis de la utilización de las tecnologías de la información y la comunicación en el seno de las asignaturas para este propósito (Briones y Lara, 2016) o en la adopción de determinados enfoques didácticos, como el modelo pedagógico basado en el enfoque histórico cultural, descrito por Molina, Silva y Cabezas (2005). Como se ha dicho, son más abundantes los estudios sobre experiencias en tipos específicos de educación: la denominada educación para el desarrollo, la educación en derechos humanos, la educación intercultural o antirracista, la feminista o la sostenibilidad ambiental.

Hay que insistir en la importancia de este tipo de educación en la formación inicial de todo tipo de profesionales, pero particularmente del profesorado, por su influencia en el desarrollo de personalidades informadas, empáticas, participativas en las cuestiones sociales más relevantes, movidas por consideraciones éticas.

Teniendo en cuenta lo hasta ahora expuesto, en este trabajo se realiza un análisis de la asignatura Educación para la paz y la igualdad, de carácter obligatorio, que se imparte en primer curso de los Grados de Maestro o Maestra en las cuatro Facultades de Educación (Palencia, Soria, Segovia y Valladolid) de la Universidad de Valladolid (UVa). Dado que el campus en el que imparten su docencia quienes escriben este artículo es el de Segovia, la investigación se centra en la asignatura que se imparte allí.

Los objetivos son los siguientes: a) analizar la valoración de la asignatura y la percepción de los y las estudiantes sobre su influencia tanto para vida personal como profesional; b) valorar los cambios producidos en la comprensión y en las actitudes mostradas ante determinadas realidades clave que se abordan en la asignatura y determinar si hay indicios de que el alumnado haya desarrollado un conocimiento crítico sobre esas realidades; c) estudiar la proyección de la asignatura en el marco institucional de la UVa y la existencia de evidencias de influencia significativa en la trayectoria académica y profesional del alumnado que ha cursado la asignatura.

\section{Método}

\section{Diseño de investigación}

El estudio se desarrolla mediante una metodología cualitativa, concretada en un estudio de casos sobre el desarrollo de la asignatura en la Facultad de Educación de Segovia. Se elige este método o enfoque - estudio de casos es un término "paraguas", según criterio de Álvarez y San Fabián (2012) - porque se pretende la comprensión de la realidad de la asignatura, su influencia y su proyección, sin olvidar el contexto en el que 
surge y se desarrolla. Además, se dan algunas de las características citadas por Rodríguez, Gil y García (1996) para la elección de este método: la unicidad, dado que se centra en una situación que tiene un carácter específico y peculiar; su carácter revelador, pues analiza una experiencia poco habitual para la investigación educativa; finalmente, la facilidad para acceder y permanecer en el caso.
Se trata de un estudio de caso intrínseco, en la clasificación de Stake (2005), pues la asignatura tiene especificidades propias y se desea alcanzar una mejor comprensión de esta.

\section{Instrumentos}

Para llevar a cabo la investigación se han utilizado diferentes instrumentos, a los cuales se han asignado códigos para efectuar el análisis de resultados (véase Tabla 1).

Tabla 1. Técnicas e instrumentos utilizados y códigos

\begin{tabular}{|c|c|c|}
\hline Técnicas e instrumentos & Tipos y participantes & Codificación \\
\hline Entrevistas & $\begin{array}{l}\text { Individuales: } 2 \text { ( } 1 \text { profesor y } 1 \text { profesora) } \\
\text { Grupales: } 6 \text { ( } 4 \text { alumnado del grado de Educación Infantil y } 2 \text { alumnado del } \\
\text { grado de Educación Primaria). }\end{array}$ & $\mathrm{EIP}_{n^{\prime}} \mathrm{EGE}_{\mathrm{n}}$ \\
\hline Cuestionarios abiertos & $\begin{array}{l}2 \text { momentos diferentes: inicio y final de la asignatura en cada curso. Desde el } \\
\text { curso 2008/2009 hasta el 2017/2018. }\end{array}$ & $\mathrm{CI}_{\mathrm{n}}($ curso $) \mathrm{CF}_{\mathrm{n}}($ curso $)$ \\
\hline Observación no participante & $\begin{array}{l}4 \text { registros de "no participante conocido", mediante cuaderno de campo: } 2 \text { de } \\
\text { una estudiante de doctorado, } 2 \text { de un docente de otra asignatura. }\end{array}$ & $\begin{array}{l}\text { OnPEst }_{n} \\
\text { OnPDoc }_{n}\end{array}$ \\
\hline Análisis de documentos & $\begin{array}{l}\text { Guías docentes de la asignatura } \\
\text { Memorias verificadas de los planes de estudio de Educación Infantil y Prima- } \\
\text { ria de la UVa } \\
\text { Plan de Organización Docente de la UVa } \\
\text { Otros documentos de la intranet de la UVa }\end{array}$ & $\begin{array}{l}\text { GD(curso) } \\
\text { MVEI, MVEP } \\
\text { POD UVa } \\
\text { Otros }\end{array}$ \\
\hline Producciones del alumnado & $\begin{array}{l}\text { Realizadas en las actividades de enseñanza-aprendizaje de la asignatura } \\
\text { desde el curso 2008/2009 hasta el curso 2017/2018. }\end{array}$ & PA(curso) \\
\hline
\end{tabular}

En el caso de las entrevistas, se han realizado dos individuales a un profesor y a una profesora de la asignatura y seis grupales a alumnas y alumnos de la asignatura, a la finalización de dos cursos académicos diferentes, 2012 y 2015, por lo que más exactamente habría que hablar no de estudiantes, sino de egresados de la asignatura. Se trata, según la clasificación en tipos enunciada por McKernan (1999), de entrevistas semiestructuradas, pues se realizan mediante un guion que contiene preguntas para todas las personas entrevistadas, pero se permite a las mismas plantear cuestiones y realizar observaciones sobre otros asuntos de interés, de manera natural durante la realización de la entrevista.

Los cuestionarios abiertos se vienen aplicando a los y las estudiantes desde la implantación de la asignatura en el curso 2008/2009 y se utilizan en dos momentos diferentes: en el comienzo de la asignatura y en el momento final de la misma. La intención es servirse de los cuestionarios como un instrumento para valorar las modificaciones en los conocimientos y en las actitudes del alumnado. En los cuestionarios se incluyen preguntas sobre conceptos o hechos y se solicitan opiniones, así como argumentaciones sobre distintas situaciones reales.

En cuanto a los registros de observación no participante han sido llevados a cabo en dos momentos diferentes y por dos personas distintas: en los cursos 2010/2011 y 2011/2012, por una estudiante de doctorado, y, en los cursos 2016/2017 y 2017/2018, por un profesor de otra asignatura. Tal y como señala Santos Guerra (1990) se trata de un "no participante conocido": el investigador o investigadora está en la acción pero no interviene en la misma. Los registros se han volcado, en forma de incidentes críticos, en los cuadernos de campo que se han utilizado por las personas citadas.

El análisis de documentos se ha realizado sobre las guías docentes de la asignatura, las memorias verificadas de los planes de estudio de Educación Infantil y Primaria de la Universidad de
Valladolid y sobre los datos extraídos de la intranet de la Universidad de Valladolid, en particular sobre el Plan de Organización Docente de la misma.

Las producciones del alumnado a las que se ha hecho referencia son las realizadas en las actividades de enseñanza-aprendizaje de la asignatura y se trata de un amplio abanico de estas, pues van desde relatos autobiográficos a propuestas didácticas del alumnado programadas para llevarse a cabo en aulas de Educación Infantil o de Educación Primaria.

\section{Procedimiento}

Para el desarrollo de la investigación se siguieron las fases generales enunciadas por Álvarez y San Fabián (2012): una fase preactiva, que ha sido replanteada a lo largo del estudio; una fase interactiva centrada en recoger, reducir y relacionar la información recogida a través de las diferentes técnicas utilizadas; y una fase postactiva o de elaboración del informe final.

La autoría de la investigación corresponde a una docente actual de la asignatura, a otra persona que también fue docente de la misma, pero en la actualidad no la imparte y a una contratada predoctoral que realiza su investigación fuera del marco de la asignatura. Como expone McKernan (1999), se ha tratado de aprovechar las ventajas de la participación en el propio caso - recogida de textos auténticos, verificación de ideas mediante observaciones empíricas, disponibilidad para una permanencia larga en el contexto estudiado,... - con la posibilidad que ofrece la investigadora no participante de no perturbar el ethos y la cultura de la asignatura con una actividad invasiva. Para preservar la credibilidad de la investigación, además del trabajo prolongado y la observación persistente, se ha utilizado la comprobación con las personas participantes y la triangulación, tanto de informantes como de técnicas y de momentos. 
En la obtención de datos se garantizó al alumnado la confidencialidad, el anonimato y el uso de la información resultante únicamente con fines investigadores. Los datos han sido analizados mediante categorías establecidas a partir de los objetivos de la investigación.

\section{Contextualización de la asignatura}

La formación del profesorado de Educación Primaria y de Educación Infantil viene regulada por la Orden ECI/3857/2007 y por la Orden ECI/3854/2007, por las que se establecen los requisitos para la verificación de los títulos universitarios oficiales que habiliten para el ejercicio de esas profesiones. Entre los objetivos y competencias que el futuro profesorado debe conseguir se establecen el análisis y el tratamiento educativo, de forma crítica, de cuestiones como la igualdad de género, la equidad, la interculturalidad, el rechazo de la discriminación y el fomento de la inclusión social y el desarrollo sostenible, el respeto a los derechos humanos y la valoración de la responsabilidad individual y colectiva en la consecución de un futuro sostenible.

Para conseguir esos objetivos y para organizar un trabajo sistemático de las competencias que vienen exigidas por la Ley 3/2007, de 22 de marzo, para la igualdad efectiva de hombres y mujeres, y la Ley 27/2005, de 30 de noviembre, de fomento de la educación y de la cultura de la paz, según se justifica en la memoria de verificación de los títulos de Grado en Maestro o Maestra de la UVa, se creó la asignatura Educación para la paz y la igualdad.

Se trata de una asignatura obligatoria, de formación básica, de 6 ECTS, que se imparte en el primer curso de ambos grados. La asignatura está organizada en 4 bloques:

- Educación para la paz y la igualdad. Conceptos básicos y estado de la cuestión.

- Educación para la paz y derechos humanos.

- Género, educación para la igualdad y currículum.

- La Educación para el desarrollo. Movimientos sociales, pobreza y globalización.

La introducción de esta en el plan de estudios no estuvo exenta de dificultades: se apoyó en la circunstancia de que la responsabilidad de la coordinación de las comisiones creadas para la elaboración y redacción de los planes de estudio recayó en personas con experiencia previa como activistas o como miembros de organizaciones comprometidas con la promoción de los derechos humanos o la cooperación internacional. Por otra parte, se realizó un trabajo sistemático de consulta con maestros o maestras en ejercicio, que refrendaron la creación de la asignatura, pese a las propuestas que formularon algunos departamentos para eliminar la misma. Se propuso que se aprovecharan experiencias ya existentes de asignaturas optativas o de libre configuración o de cursos y actividades relativos todos ellos a las cuestiones abordadas por la asignatura y, de ese modo, la docencia fue atribuida a unidades docentes con realizaciones en ese ámbito. Es decir, no se asignó la asignatura a una determinada área, sino que, en cada centro, la asignatura fue adscrita al área con experiencias previas en estos ámbitos.

Entre los objetivos de la asignatura, establecidos en las memorias verificadas, merece destacarse el primero:

Promover el desarrollo de un compromiso ético en su configuración como profesional, compromiso que debe potenciar la idea de educación integral, con actitudes criticas y responsables; garantizando la igualdad efectiva de mujeres y hombres, la igualdad de oportunidades, la accesibilidad universal de las personas con dis- capacidad y los valores propios de una cultura de la paz y de los valores democráticos. (MVEI, MVEP).

\section{Análisis de resultados}

\section{Valoración de la asignatura y percepción de su influencia}

La asignatura recibe, de forma muy mayoritaria, una valoración que oscila entre positiva y muy positiva, con predominio de la última, según se desprende de los cuestionarios realizados, con carácter anónimo, al final de la asignatura:

Nunca en toda mi historia escolar había recibido una asignatura así [...] He aprendido que es posible educar los valores y me ha permitido crearme mi propia idea de cómo está el mundo. En general, la asignatura y su desarrollo han sido excelentes. $\left(\mathrm{CP}_{19}[16 / 17]\right)$.

Tengo que reconocer que es la asignatura que más me ha gustado. Siempre he salido con algo nuevo aprendido. En realidad, me siento muy orgullosa de lo que he aprendido y me parece una asignatura imprescindible. No imagino cómo se puede estudiar Magisterio sin esta asignatura. $\left(\mathrm{CI}_{22}[15 / 16]\right)$.

En las entrevistas grupales también se pone de manifiesto esa valoración mayoritaria de la asignatura, con una tendencia frecuente a subrayar la apertura a una nueva visión de la situación mundial, antes desconocida para un grupo muy nutrido de estudiantes. Destaca también la comprensión y la reflexión sobre la realidad realizada por una buena proporción del alumnado:

A mí, en particular, la asignatura me pareció bastante coherente: gracias a ella he visto $y$ he comprendido otras formas de vida que sirven para transformar y mejorar el mundo y conseguir, así, una sociedad igualitaria y sin discriminaciones. Las sesiones y el trabajo se enfocaban, precisamente, a eso. $\left(\mathrm{EGE}_{2)}\right.$.

La asignatura me ha parecido muy positiva, ya que nos ha hecho ver diferentes puntos de vista a los que nosotros vemos o pensamos y también nos refleja la realidad de muchas personas día a día y sólo por pensar que son de otros países, por ejemplo, no hacemos nada por ellos. También me ha parecido importante recordar los Objetivos del Milenio, ya que nunca había profundizado en ellos y creo que es fundamental su lucha y abolición. $\left(\mathrm{EGE}_{1}\right)$.

Algunas estudiantes rescatan, en esa evaluación favorable que realizan de la asignatura, aspectos esenciales y muestran que se han conseguido los objetivos, pues aparecen testimonios de una transformación profunda de la persona:

He aprendido a ver el mundo de manera diferente y cambiar comportamientos hacia las demás personas y he aprendido tanto a respetarme a mí como al mundo. Lo que más destacaría sin ninguna ha sido las reflexiones que me ha llevado a hacer porque realmente puedo decir que he cambiado mi imagen de muchas cosas totalmente y me fijo mucho más en otras que por ejemplo antes no lo daba la suficiente importancia. Me hacía mucha falta. $\left(\mathrm{CP}_{2}[17 / 18]\right)$.

No obstante, si detenemos la mirada en las opiniones veremos que esta valoración favorable se basa, con frecuencia, en elementos que no forman parte del corazón radical de la asignatura, de su esencia constitutiva, sino que se refieren a los métodos utilizados o las actividades desarrolladas. Aparece una visión más "ligera" de lo abordado en la asignatura:

Aspectos positivos son que aparte de dar la teoría, veíamos vídeos para entender la teoría, leíamos cuentos, hacíamos asambleas 
para participar todos, íbamos al gimnasio y realizábamos juegos. Este cuatrimestre me ha gustado mucho porque hemos aplicado técnicas que nunca las había aplicado. $\left(\mathrm{CP}_{21}[17 / 18]\right)$.

En todo caso, la valoración de asignatura se mantiene en términos muy positivos a lo largo de los cursos y se estima fundamental tanto para la formación personal como para la profesional como docentes, de manera que es considerada como una asignatura importante e influyente en la formación. Llama la atención que para justificar este aprecio se recurra tanto a los temas abordados, como a las actividades realizadas, a los métodos empleados o a la posibilidad que se ofrece en la asignatura de visitar colegios y desarrollar actividades en los mismos. Los objetivos de la asignatura sólo son enunciados por una parte reducida de personas.

\section{Cambios producidos en la comprensión y en las actitudes ante realidades clave}

Si la valoración de la asignatura es alta y la opinión general es que se trata de una asignatura con influencia formativa, no se puede afirmar lo mismo, en líneas generales, sobre la profundidad de las modificaciones en la comprensión de la realidad global en la que vivimos.

Es cierto que aparecen, en bastantes participantes, recuerdos de momentos clave que marcan un cambio, un desequilibrio en el modo de ver la realidad y ese cambio es perdurable. Por ejemplo, una de las egresadas afirma en una entrevista:

A mí, entre otras cosas, se me quedó grabado cuando estábamos trabajando en lo de la igualdad de género, y nos dijo el profesor: ¿Creéis que...? ¿Cómo lo dijo...? ¿Creéis que el hombre tiene que ayudar a la mujer en las tareas del hogar? Y todos: sí, sí, sí, sí, sí... $Y$ cogió y dijo: pues mal...mal, porque no se trata de que uno ayude al otro, ni mucho menos que el hombre ayude a la mujer, sino de que los dos, los dos colaboren... Entonces, yo nunca me lo había planteado así... Claro es que... lo que nos dijo que, el propio contexto a veces con etiquetas y esas cosas... ( $\left.\mathrm{EGE}_{3}\right)$.

Hay algunas respuestas que denotan que no se ha producido ese necesario desaprendizaje que se reflejaba en el comentario anterior, esa reflexión sobre las propias concepciones y sobre la mirada a la realidad. La visión de esas realidades podría considerarse poco profunda, casi distraída o con una actitud que resbala sobre los ángulos de las situaciones que marcan la vida de los seres humanos:

Creo que la asignatura ha sido entretenida, ya que hemos realizado juegos, leído cuentos, vídeos que nos ayudan a entender mejor la asignatura, etc. por lo tanto considero que ha sido interesante, que he aprendido mucho respecto a diversos temas, como interculturalidad, derechos humanos y de los niños, etc. $\left(\mathrm{CP}_{20}[17 / 18]\right)$.

La formación de personas críticas pasa por el abandono de la pasividad y del conformismo, por el ejercicio del pensamiento crítico en todas las ocasiones y hay situaciones en la asignatura que muestran lo que queda por conseguir, además de indicar que las actitudes no han variado sustancialmente:

Hoy en clase se ha realizado un ejercicio de educación para la desobediencia: la profesora ha entrado, diciendo que había un examen. Ante la sorpresa colectiva, ha seguido dando consignas absurdas de cómo poner el nombre, colocar el bolígrafo y quitar el material del compañero. Tras el desconcierto inicial, la clase, sin reparar siquiera en que está en una asignatura que enfatiza el pensamiento crítico, ha comenzado a obedecer, a mostrar una actitud sumisa, con alguna excepción. No se han planteado lo arbitrario de la conducta de la profesora. (OnPDoc $)$.

Puede afirmarse que los cambios más visibles son los producidos en los conocimientos previos sobre educación para la paz y la igualdad. Si nos detenemos en la igualdad de género otra vez, nos encontraremos con dos cuestiones planteadas en el cuestionario inicial, que constituyen un buen indicador de la magnitud del aprendizaje realizado: se pregunta si la escuela mixta y la coeducativa son lo mismo y si el feminismo es lo contrario del machismo. Con respecto a la primera cuestión, en el curso 2011/2012, el 49,2\% no contesta o admite no saberlo y el $23,3 \%$ afirma que no son lo mismo pero no llega a explicar en qué se diferencian o da una explicación muy poco concreta; tan sólo el 11,6 \% explica con cierta claridad la diferencia, relacionándola con la igualdad de género. En el cuestionario final, este último tipo de respuestas alcanza el $65,4 \%$, pero hay un inquietante $22,8 \%$ que sigue sin explicar la distinción con una mínima claridad. Y encontramos, también, un 11,6\% que sigue sosteniendo, tras el paso por la asignatura, que el feminismo es lo contrario del machismo, porcentaje que en el cuestionario inicial se elevaba a más del $40 \%$.

En cualquier caso, se ve una evolución notable en algunas cuestiones. Por ejemplo, se realizó un ejercicio en el que, a partir de un relato, había que reflexionar sobre las causas del hambre en el mundo. En uno de los grupos, se realizó antes de impartir el bloque relativo a la educación para el desarrollo; en el otro, tras ser impartido este bloque. Las explicaciones sobre el origen del hambre y las alternativas para enfrentarlo muestran bastantes diferencias: proponer un aumento en la producción de alimentos o el progreso tecnológico en forma de maquinaria enviada a esos países o adoptar visiones paternalistas - no son capaces de arreglárselas solos fuera de su entorno - frente al otro grupo (el que ha sido formado en educación para el desarrollo) que señala la interdependencia de las políticas, la especulación financiera sobre las materias primas o la pobreza estructural (PA[14/15]). Una descripción detallada de estos resultados puede verse en Martínez, Monjas y Torrego (2016).

Proyección de la asignatura en el marco institucional de la universidad e influencias en la trayectoria académica y profesional del alumnado.

La proyección institucional de la asignatura conoce circunstancias bien diferentes. Por un lado, puede observarse como durante el proceso de verificación y en el proceso de acreditación del título, la agencia señala como uno de los puntos fuertes de los títulos, la creación de la asignatura. Por otra parte, cuando, desde el curso 2010/2011, se suceden recortes presupuestarios que afectan a la plantilla de la UVa, esta asignatura es una de las primeras en sufrir cambios en la adscripción del profesorado: pasan a impartirla personas que no han tenido vinculación con los bloques de esta, con la intención de aprovechar profesorado de departamentos con menor carga docente. Así, en Segovia, en Educación Primaria, la docencia se asigna a un profesor del "área de Análisis Geográfico Regional” (POD). Esta modificación en la asignación de la asignatura no se ha revertido en la actualidad.

La asignatura tiene una incidencia innegable en los temas elegidos para desarrollar trabajos de fin de grado o de máster e incluso tesis doctorales. Así, una consulta en UVaDoc, el repositorio virtual en el que se alojan estos trabajos arroja el resultado de que al menos 59 trabajos, por el título, corresponden 
a los bloques de contenido de la asignatura. La educación para el desarrollo y las cuestiones relacionadas con el género, son las temáticas más abordadas. Incluso 4 tesis doctorales, 2 de ellas ya defendidas, se centran en la asignatura. Hay que considerar estos datos en el contexto de un centro pequeño, como es la Facultad de Educación de Segovia.

Otro indicador de la incidencia puede obtenerse, indirectamente, a través del activismo desarrollo por el alumnado o de su participación en iniciativas sociales, políticas y solidarias. Con respecto a este indicador, puede valorarse como incipiente la influencia. Así, una parte del alumnado se ha mostrado activo en las actividades promovidas en la universidad por dos ONGD, que actúan en la República Dominicana y en Ghana, respectivamente, y se ha movilizado en defensa de la universidad pública con protestas, manifestaciones y encierros, sobre todo en el curso 2011/2012, a través de Segovia en Vela, una iniciativa constituida para ese fin. También han participado en acciones contra la violencia de género y el rechazo del sexismo. No puede afirmarse que estas movilizaciones sean masivas, pues buena parte de los y las estudiantes no ha intervenido en las mismas.

Uno de los profesores de la asignatura, en una de las entrevistas, muestra su insatisfacción por este grado de incidencia conseguida. La afirmación es, a la vez de la constatación de un logro insuficiente, una muestra de implicación con lo que enseña:

Yo lo que sí que sé es que esta asignatura es..., deberíamos esforzarnos por convertirla en un espacio de conflicto, damos poca guerra, a ver si me explico, ...no puede ser una asignatura más..., ya sé que tiene sus seminarios, sus prácticas, su teoría, su tal, su matrícula como todas las demás, pero tiene que ser diferente, incluso debería ser de alguna manera conflictiva para la sociedad en la que vivimos, la universidad en la que vivimos, conflictiva porque realmente plantee, someta a discusión, las cosas que se están haciendo en cada momento, de manera que no las demos por sentadas, por válidas, por naturalizadas. $\left(\mathrm{EIP}_{1}\right)$.

\section{Discusión y conclusiones}

La asignatura Educación para la paz y la igualdad es la única existente con esa denominación en las universidades españolas. Sus objetivos son ambiciosos, pues pretende formar docentes que contribuyan con su formación crítica y ética, con su testimonio, a que los educandos y educandas, se vayan transformando en lo que Freire (1994) llama presencias notables en el mundo, de manera que se consideren y se conviertan en protagonistas de la historia. Según se pone de manifiesto en los cuestionarios iniciales y en las producciones del alumnado, es frecuente que cuando comiencen los estudios de Magisterio, la realidad se considere como algo estático, como naturalizada, sin plantearse la posibilidad de modificación o como completamente ajena a su experiencia existencial. Esa es la barrera que pretende contribuir a romper la asignatura.

Hemos podido constatar que los logros de la asignatura son parciales. Se proyecta el desarrollo de conocimientos, de actitudes y también de motivaciones para actuar. Al final de la asignatura se produce, de forma generalizada, un aumento de sus conocimientos sobre las causas y consecuencias de las situaciones globales de injusticia y, también, un incremento de la consciencia de que es necesario actuar. Se instala, pues, un discurso que podríamos considerar "correcto" sobre la realidad global. Es una situación similar a la que se ha encontrado en otros estudios, como el realizado por Martínez y Zurita, (2011) con respecto a las actitudes del alumnado de Magisterio hacia la interculturalidad.

Nuestro estudio confirma la realidad encontrada por Martínez-Scott (2014), que en su tesis doctoral, realizada en torno a la educación para el desarrollo que se promueve desde la asignatura, constata que la mayoría del alumnado no abandona la actitud pasiva generalizada y que, pese a manifestar su consciencia de que es necesario actuar para transformar el planeta y el modo de vida de sus habitantes, se refugia en la falta de información, en la ausencia de valores en la sociedad, en la desconfianza hacia organizaciones o personas o, directamente, declina en los gobiernos toda la responsabilidad del cambio. Puede afirmarse que en una buena parte de las y los estudiantes aparece una "solidaridad de demanda", que debe solicitarse a las instancias con poder. Se trata no sólo de una visión desmovilizadora, sino también poco ética - no se asumen las propias responsabilidades - y poco crítica - no parece razonable que sea el poder que causa la injusticia quien se empeñe en acabar con ella - o bastante ingenua.

Por otra parte, la valoración que se realiza de la asignatura es muy positiva y se la considera útil, tanto desde el punto de vista profesional como personal. En el primero de los ámbitos se valora la formación metodológica proporcionada y las estrategias didácticas aprendidas, como el tratamiento educativo del conflicto, el descubrimiento de las tertulias literarias dialógicas, la adopción del enfoque socioafectivo o los juegos y dinámicas de cooperación. En el segundo, un grupo numeroso de estudiantes señala haber sido consciente de los prejuicios y estereotipos que mantenía en cuestiones relativas a la diversidad cultural, al género o a la cooperación internacional y también es muy común la respuesta de que la asignatura les ha abierto los ojos, les ha proporcionado otra mirada hacia la realidad.

La investigación pone de manifiesto lo ya señalado por otros estudios (Morales, Trianes e Infante, 2013): la relevancia que tiene la evaluación de valores éticos y sociales y de las actitudes que conducen hacia la movilización de conductas prosociales/solidarias y su relación con la adquisición de competencias solidarias.

En los planes de estudio de la mayoría de las universidades se ha decidido no incluir contenidos específicos de educación para la paz y de educación para la igualdad, se ha optado por realizar la formación del profesorado tal y como es señalado por González-Gil, Martín-Pastor y Orgaz (2017): de manera transversal y holística. El problema es que, en muchas de ellas, no hay ningún tratamiento sistemático de estos ejes: lo transversal se difumina o se atraviesa, en el sentido de que otros contenidos impiden el paso a la formación crítica y emancipadora.

Las dificultades en organizar un pensamiento crítico y en movilizar a los futuros maestros y maestras nos llevan a considerar la necesidad de una repolitización de la educación, en el sentido etimológico del término: la escolaridad, desde sus inicios, debería preocuparse por las cuestiones comunes, por la sociedad en que vivimos, y debería tener incidencia en su transformación, apostando por una educación democrática.

La Educación para la paz y la igualdad navega contra corriente: el ambiente universitario no es el que ha generado el optimismo de la ética y del compromiso social; de ahí que los resultados muestren luces y sombras. En cualquier caso, la educación no puede concebirse sin una visión de un futuro mejor y más digno, presidido por el bien común, y a ello contribuye esta asignatura. Quienes participamos en ella soñamos con aportar un grano de arena al surgimiento de una generación de docentes que eduquen para la desobediencia.

\section{Referencias bibliográficas}

Aguilar, C. (2013). Género y formación crítica del profesorado: una tarea urgente y pendiente. Revista Interuniversitaria de Formación del Profesorado, 78(27,3), 23-34. 
Álvarez, C., y San Fabián, J. L. (2012). La elección del estudio de caso en investigación educativa. Gazeta de Antropología, 28(1).

Banco Mundial (2003). Construir Sociedades de Conocimiento: Nuevos Desafíos para la Educación Terciaria. Washington: Autor.

Baños, J. (2013). Democracia y ética: el republicanismo cívico de Hannah Arendt. Estudios políticos, 30, 79-103.

Boff, L. (2001). Ética planetaria desde el Gran Sur. Madrid: Trotta

Bolívar, A. (2005). El lugar de la ética profesional en la formación universitaria. Revista mexicana de investigación educativa, 10(24), 93-123.

Boni, A., y Gasper, D. (2011). La Universidad como debiera ser. Propuestas desde el desarrollo humano para repensar la calidad de la Universidad. Sistema, 220-221, 93-109.

Briones E., y Lara, L. (2016). Teaching ethics in the university through multicultural online dialogue. Comunicar, 24(47), 99107.

Calvo, A., y Rodríguez-Hoyos, C., y Haya, I. (2015). Con motivo aparente. La Universidad a debate. Revista Interuniversitaria de Formación del Profesorado, 82, 17-33.

Cecchi, N., Pérez, D., y Sanllorenti, P. (2013). El compromiso social de la Universidad Latinoamericana del Siglo XXI: entre el debate y la acción. Buenos Aires: IEC - CONADU.

Consejo de la Unión Europea (2007). Council Resolution on modernising universities for Europe's competitiveness in a global knowledge economy. Recuperado de http://register.consilium. europa.eu/pdf/en/07/st16/st16096-re01.en07.pdf

Del Río, A., y Celorio, G. (Coords.). (2018). Hacia una Universidad socialmente comprometida. Vías estratégicas para su integración en la Universidad del País Vasco (UPV/EHU). Bilbao: Hegoa-EHU.

Freire, P. (1994). Cartas a quien pretende enseñar. México: Siglo XXI.

González-Gil, F., Martín-Pastor, E., y Orgaz, B. (2013). ¿Están los futuros profesores formados en inclusión?: Validación de un cuestionario de evaluación. Aula Abierta, 46, 33-40.

Ibarrola-García, S., y Artuch, R. (2016). La docencia en la universidad y el compromiso social y educativo. Contextos educativos, 19, 105-120.

Jover, G., López, E., y Quiroga, P. (2011). La universidad como espacio cívico: valoración estudiantil de las modalidades de participación política universitaria. Revista de Educación, número extraordinario, 69-91.

Manzano, V. (2011). La Universidad Comprometida. Barcelona: Hipatia.

Manzano, V., y Torrego, L. (2009). Tres modelos para la universidad. Revista de Educación, 350, 477-489.

Martínez-Martín, M. (2006). Formación para la ciudadanía y educación superior. Revista Iberoamericana de Educación (OEI), 42, 85-102.
Martínez, A. y Zurita, F. (2011). Actitudes y percepciones del alumnado de Magisterio en la especialidad de Educación Primaria ante los procesos migratorios. Aula Abierta, 39(2), 91-102.

Martínez, M., Buxarrais, M. R., y Esteban, F. (2002). La universidad como espacio de aprendizaje ético. Revista Iberoamericana de Educación (OEI), 2002, 29, 17-43.

Martínez, S., Monjas, R., y Torrego, L. (2017). Hunger and prejudice. A study of development education in teachers training. Proccedia Social and Behavioral Sciences, 237, 950-955.

Martínez-Scott, S. (2014). La educación para el desarrollo en la formación inicial del profesorado. Estudio de casos en la asignatura Educación para la paz y la igualdad. Tesis doctoral, Universidad de Valladolid Recuperado de http://uvadoc.uva.es/bitstream/10324/5691/1/TESIS563-140728.pdf

McKernan, J. (1999). Investigación-acción y curriculum. Madrid: Morata.

Molina, A.T., Silva, F.E., y Cabezas, C.A. (2005). Concepciones teóricas y metodológicas para la implementación de un modelo pedagógico para la formación de valores en estudiantes universitarios. Estudios Pedagógicos, XXXI (1), 79-95.

Morales, F. M., Trianes, V., y Infante, L. (2013). Perfiles de valores éticos en estudiantes universitarios. Aula Abierta, 41(2), 55-66.

Muhr, T. (2010). Counter-hegemonic regionalism and higher education for all: Venezuela and the ALBA. Globalization, Societies and Education, 8(1), 39-57.

Organización para la Cooperación Económica Europea (2007). Higher Education and Regions: Globally Competitive, Locally Engaged. París: Autor.

Petrova, E. (2010). Democratic Society and Moral Education. Procedia - Social and Behavioral Sciences, 2, 5635-5640.

Rodríguez, G., Gil, J., y García, E. (1996). Metodología de la investigación cualitativa. Málaga: Aljibe.

Santos Guerra, M. Á. (1990). Hacer visible lo cotidiano: Teoría y práctica de la evaluación cualitativa de los centros escolares. Madrid: Akal.

Santos, M.A., Sotelino, A., y Lorenzo, M. (Eds.). (2016). Aprendizaje-Servicio e innovación en la universidad. Santiago de Compostela: Universidad de Santiago de Compostela.

Stake, R. E. (2005). Investigación con estudio de casos. Madrid: Morata.

UNESCO (1998). Conferencia Mundial sobre la Educación Superior. La educación superior en el siglo XXI. Visión y acción. (Tomo I). París: El autor.

Vallaeys, F. (2014). A responsabilidade social universitária: um novo modelo universitário contra a mercantilização. Revista iberoamericana de educación superior, 5(12), 105-117. 
